\title{
Testing the Universality of Entropic Segregation at Polymer Surfaces
}

\author{
P. Mahmoudi, ${ }^{\dagger}$ W.S.R. Forrest, ${ }^{\ddagger}$ T.M. Beardsley, ${ }^{\ddagger}$ and M.W. Matsen ${ }^{*, \dagger},+, \uparrow$ \\ $\dagger$ Department of Chemical Engineering, University of Waterloo, Waterloo, Ontario, Canada \\ $\ddagger$ Department of Physics \& Astronomy, University of Waterloo, Waterloo, Ontario, Canada \\ \Waterloo Institute for Nanotechnology, University of Waterloo, Waterloo, Ontario, \\ Canada \\ E-mail: mwmatsen@uwaterloo.ca
}

\begin{abstract}
This study addresses entropic segregation effects at the surfaces of monodisperse and bidisperse melts. For the monodisperse melts, we focus on the segregation of chain ends to the surface, and for the bidisperse melts, we examine the segregation of short polymers to the surface. Universal shapes have been predicted for their concentration profiles, but the derivations rely on the mean-field approximation, which only treats the excluded-volume interactions in an approximate manner. To test whether or not the predictions hold up when the polymers are rigorously prevented from overlapping, we compare mean-field calculations with Monte Carlo simulations performed on the exact same model. Apart from a significant increase in the statistical segment length, the rigorous enforcement of excluded-volume interactions has a relatively small effect on the mean-field predictions. In particular, the universal profiles predicted by mean-field theory are found to be accurate.
\end{abstract}




\section{Introduction}

Polymer melts are not only commercially important, they also represent one of the most fundamental systems in soft condensed matter physics. Their equilibrium properties are of particular interest. Fortunately, the excluded-volume interactions that would potentially complicate the behavior are screened, ${ }^{1}$ and consequently polymers in the bulk exhibit random-walk statistics with an average end-to-end length of $a \sqrt{N}$, where $N$ is the number of segments per polymer and $a$ is the statistical segment length. The next issue is how the polymer statistics are affected by a surface, but this also turns out to be surprisingly simple, at least, to a first approximation. Silberberg ${ }^{2}$ has argued that surfaces just behave like reflecting boundaries. This rationalization largely accounts for the distribution of polymer conformations in the surface region. ${ }^{3,4}$

One implication of the Silberberg argument is that the distribution of any particular segment, such as an end segment, maintains a uniform bulk density all the way to the surface, in the absence of any enthalpic preference. However, simulations ${ }^{5-11}$ have found an entropic enrichment of chain ends at the surface, followed by a long-range depletion. This effect is captured by mean-field calculations, ${ }^{12-14}$ at least qualitatively. The segregation occurs due to violations in the assumptions used by Silberberg, namely a sharp step-like concentration profile and the absence of an energy penalty for folding polymer chains. ${ }^{14}$ Linear-response theory finds that the amplitude of the enrichment is dependent upon the details of the model, but that the compensating depletion exhibits a universal shape with a range proportional to $a \sqrt{N}$, regardless of model details. ${ }^{13}$

In the case of polydisperse melts, the chain-end segregation causes an enrichment of short polymers near the surface, since they have more ends per unit volume. This has yet to be demonstrated by simulation, but it has been predicted by mean-field theory. Hariharan et

al. ${ }^{15}$ did so for a bidisperse melt of short and long polymers. They investigated both the amplitude and the range of the enrichment. Van der Gucht et al. ${ }^{16}$ extended the meanfield calculations to polydisperse melts with multiple chain lengths. They found that the 
integrated excess of polymers of polymerization $N$ obeyed a strikingly simple relationship,

$$
\theta_{N} \propto \bar{\phi}_{N}\left(1-\frac{N}{N_{w}}\right)
$$

where $\bar{\phi}_{N}$ is the bulk volume fraction of the polymers and $N_{w}$ is the weight-average polymerization. This dependence has since been derived using linear-response theory. ${ }^{17,18}$ The theory predicts universal concentration profiles for the various polymerizations of the melt, which in turn implies that the functional form of eq 1 is universal. The amplitude of the effect is directly related to that of the chain-end segregation, and thus it again depends on model details.

Universality is a powerful property implying that all systems, experimental as well as theoretical, exhibit quantitatively equivalent behavior. However, we need to remember that these predictions of universality originate from linear-response theory, which relies upon the mean-field approximation. Therefore, it remains uncertain how accurate they actually are. It was previously pointed out that the excluded-volume interactions, which are neglected in mean-field theory, violate Silberberg's assumption that polymer configurations can be folded without incurring an energy penalty. ${ }^{14}$ The implication is that the mean-field predictions may be considerably inaccurate. The simplest way of assessing this is by comparing with simulation. However, as of yet, there have been no simulations examining surface segregation for polydisperse melts, and the simulations on monodisperse melts have not closely examined the long-range depletion of ends.

Here, we perform Monte Carlo (MC) simulations for monodisperse and bidisperse melts. The results are directly compared with mean-field theory for the exact same model. This provides the first quantitative assessment of how reliable mean-field theory is in treating entropic surface segregation, and in particular how accurate its universal predictions are. 


\section{Monte Carlo Simulations}

In the interest of accurate statistics, our MC simulations employ a simple model, ${ }^{19}$ whereby the polymers are restricted to an fcc lattice with a maximum of one monomer per lattice site and bonded monomers occupying nearest-neighbor sites. The fcc lattice is created by taking a simple-cubic $L \times L \times L$ lattice and deleting every second site, leaving a total of $L^{3} / 2$ sites. To allow room for the polymers to move, the lattice is only filled to $80 \%$ occupancy, which corresponds to an average monomer density of $\rho_{0} \approx 0.8 \sqrt{2} / b^{3}$, where $b$ is the bond length (i.e., the lattice spacing). At this density, the statistical segment length is known to be $a=1.233 b .^{20}$

Rather than relying on cohesive interactions between monomers to create surfaces, we simply constrain the polymers between two hard walls in the (001) direction. This is done by applying periodic boundary conditions in the $x$ and $y$ directions, while restricting the polymers between $z=0$ and $(L-1) \Delta z$, where $\Delta z=b / \sqrt{2}$ is the spacing between lattice planes. We also apply an external field, $w(z)$, so as to create a uniform polymer concentration up to the edge of the walls. The energy of the field is

$$
E=\sum_{z} \hat{m}(z) w(z)
$$

and the polymer concentration is defined as

$$
\hat{\phi}(z) \equiv \frac{2 \hat{m}(z)}{\rho_{0} L^{2}}
$$

where $\hat{m}(z)$ is the total number of monomers in the lattice layer at $z$. This definition of $\hat{\phi}(z)$ is such that its thermodynamic average, $\phi(z) \equiv\langle\hat{\phi}(z)\rangle$, equals one, once the field has been appropriately adjusted. Given the uniform polymer concentration, the energy of monomermonomer interactions would be relatively constant, and thus such interactions should have a negligible effect on the simulations. For this reason, we do not include interactions between 
the monomers, apart from the constraints that prevent the overlap of monomers and that link the bonded monomers together.

Monte Carlo simulations of this model are performed in the canonical ensemble, where the number of molecules remains fixed. The Monte Carlo steps (MCS) involve selecting a molecule at random, and attempting with equal probability either a slithering snake or crankshaft move. ${ }^{19}$ Moves are accepted or rejected using the standard Metropolis criterion. In order to fill the lattice, the polymers are initially placed on the lattice in extended configurations. Therefore, we generally start with $10^{6}$ MCS per monomer to allow the polymers to adopt coiled configurations. Following that, we perform a further $10^{5}$ MCS per monomer, where we adjust the field after each MCS per monomer using a simple-mixing iteration

$$
w^{(k+1)}(z)=w^{(k)}(z)+\lambda(\hat{\phi}(z)-1)
$$

with a mixing parameter of $\lambda=0.01$. This value is sufficiently large to provide a reasonable rate of convergence, but not so large as to cause an instability. Once the system is well equilibrated and the field has saturated, we fix the field and collect statistics for typically $10^{7}$ MCS per monomer. Thermodynamic averages are evaluated by sampling observables once every 40 MCS per monomer.

Our monodisperse melts consist of $n$ polymers with polymerization $N$. For this system, we sample the dimensionless concentration of end monomers,

$$
\hat{\phi}_{e}(z) \equiv \frac{N \hat{m}_{e}(z)}{\rho_{0} L^{2}}
$$

where $\hat{m}_{e}(z)$ is the total number of end monomers at $z$. This definition is such that the average concentration, $\phi_{e}(z)=\left\langle\hat{\phi}_{e}(z)\right\rangle$, becomes one in the bulk, and thus the excess concentration of end monomers is just

$$
\delta \phi_{e}(z) \equiv \phi_{e}(z)-1
$$


For bidisperse melts, we fill the lattice with $n_{s}$ short and $n_{l}$ long polymers, consisting of $N_{s}$ and $N_{l}$ monomers, respectively. The dimensionless concentrations of the two polymerizations are given by

$$
\hat{\phi}_{\nu}(z) \equiv \frac{2 \hat{m}_{\nu}(z)}{\rho_{0} L^{2}}
$$

where $\hat{m}_{\nu}(z)$ is the total number of monomers at $z$ belonging to the $\nu$-type polymers $(\nu=s$ or $l$ ). We focus on the excess concentration of short polymers defined by

$$
\delta \phi_{s}(z) \equiv \phi_{s}(z)-\bar{\phi}_{s}
$$

where $\phi_{s}(z)=\left\langle\hat{\phi}_{s}(z)\right\rangle$ and $\bar{\phi}_{s}$ is the average bulk concentration in the middle of the simulation box. From that, we obtain the integrated excess

$$
\theta_{s} \equiv \rho_{0} \sum_{z} \delta \phi_{s}(z) \Delta z
$$

by summing from $z=0$ to the middle of the box (i.e., where $\left.\delta \phi_{s}(z)=0\right)$.

Naturally, the size of our simulation box, $L$, has to be sufficient to attain bulk behavior in the middle, but not so large that the simulations are unnecessarily slow. Our choice of $L$ is guided by the mean-field calculations, which will be described in the next section. For bidisperse melts, we generally want appropriate values of $n_{s}$ and $n_{l}$ to target a particular bulk composition, $\bar{\phi}_{s}$, while providing an overall monomer concentration of $\rho_{0}=0.8 \sqrt{2} / b^{3}$. It will turn out that the mean-field calculations are sufficiently accurate to provide reasonable estimates of these values. Nevertheless, we typically tweak the $n_{\nu}$ values and rerun the simulations a second time to obtain $\bar{\phi}_{s}$ values that are well within one percent of our targets.

\section{Mean-Field Theory}

The mean-field theory for lattice models has been developed by Scheutjens and Fleer. ${ }^{21,22}$ It also involves a field, but this time $w(z)$ accounts for all non-bonded interactions. The 
main computational step is to calculate a partition function, $G_{i}(z)$, for a chain fragment of $i$ monomers with one end fixed at $z$. It is evaluated by applying the recursive relation

$$
G_{i+1}(z)=h(z) \sum_{Z} g(Z) G_{i}(z-Z)
$$

starting from $G_{1}(z)=h(z)$, where $h(z) \equiv \exp \left(-w(z) / k_{B} T\right)$. The function $g(Z)$ is the fraction of nearest-neighbor sites a monomer at $z$ has in the lattice layer at $z+Z$; for (001) layers of an fcc lattice, $g(0)=g( \pm 1)=1 / 3$ and zero otherwise. Equation 10 is solved with $h(z)=0$ for $z<0$ and a reflecting boundary at the large- $z$ end of our system. Naturally, the system size has to be sufficiently large that we obtain bulk behavior at large $z$. Just as in the simulations, $w(z)$ is adjusted using the same simple-mixing iteration, eq 4 , but with the instantaneous concentration, $\hat{\phi}(z)$, replaced by the average concentration, $\phi(z)$. For convenience, we adjust the field by an additive constant so that it is zero in the bulk (i.e., at large $z$ ).

In the case of monodisperse melts, the average polymer concentration is given by

$$
\phi(z)=\frac{1}{N h(z)} \sum_{i=1}^{N} G_{i}(z) G_{N+1-i}(z)
$$

and the average end-monomer concentration is simply

$$
\phi_{e}(z)=G_{N}(z)
$$

For bidisperse melts, the total concentration is $\phi(z)=\phi_{s}(z)+\phi_{l}(z)$, where the concentrations of the two polymerizations are evaluated using

$$
\phi_{\nu}(z)=\frac{\bar{\phi}_{\nu}}{N_{\nu} h(z)} \sum_{i=1}^{N_{\nu}} G_{i}(z) G_{N_{\nu}+1-i}(z)
$$




\section{Linear-Response Theory}

Linear-response theory is an approximation of mean-field theory that becomes increasingly accurate for large polymerizations. The application to our lattice model is a straightforward adaption of the calculations in refs 14 and 18. The first step involves solving the mean-field theory for infinitely long polymers, where

$$
G_{\infty}(z)=h_{\infty}(z) \sum_{Z} g(Z) G_{\infty}(z-Z)
$$

with $h_{\infty}(z)$ adjusted so that

$$
\frac{G_{\infty}^{2}(z)}{h_{\infty}(z)}=1
$$

From the solution, the amplitude of the entropic segregation is ${ }^{18}$

$$
A=\frac{1}{\sqrt{2}} \sum_{z}\left[G_{\infty}(z)-1\right]=0.1895
$$

The long-range depletion of end monomers in a monodisperse melt is given by ${ }^{13}$

$$
\delta \phi_{e}(z) \approx \frac{A}{\sqrt{N}} B\left(\frac{z}{a \sqrt{N}}\right)
$$

where

$$
B(\zeta)=\sqrt{\frac{6}{\pi}} \exp \left(-\frac{3}{2} \zeta^{2}\right)-4 b(\zeta)
$$

is a universal function. The later part of the function is obtained by performing an inverse Fourier transform,

$$
b(\zeta) \equiv \frac{1}{2 \pi} \int_{-\infty}^{\infty} \frac{s_{e}^{2}(x)}{s_{\phi}(x)} e^{i k_{\zeta} \zeta} d k_{\zeta}
$$

where $s_{\phi}(x)=2\left(e^{-x}+x-1\right) / x^{2}$ is the Debye function, $s_{e}(x)=\left(1-e^{-x}\right) / x$ is a Debye-like function, and $x=k_{\zeta}^{2} / 6$. 
For bidisperse melts, the excess concentration of short polymers is approximated by ${ }^{18}$

$$
\delta \phi_{s}(z) \approx \frac{A}{\sqrt{N_{s}}} C\left(\frac{z}{a \sqrt{N_{s}}}\right)
$$

where

$$
C(\zeta)=4 \bar{\phi}_{s}\left(\sqrt{\frac{6}{\pi}} f(\zeta)-c(\zeta)\right)
$$

This universal function is made up of two parts,

$$
f(\zeta) \equiv \int_{0}^{1} \frac{1}{2 \sqrt{s}} \exp \left(-\frac{3}{2 s} \zeta^{2}\right) d s
$$

and

$$
c(\zeta) \equiv \frac{1}{2 \pi} \int_{-\infty}^{\infty} \frac{s_{\phi}(x)\left[s_{e}(x)+\beta s_{e}(\alpha x)\right]}{s_{\phi}(x)+\alpha \beta s_{\phi}(\alpha x)} e^{i k_{\zeta} \zeta} d k_{\zeta}
$$

which is a function of $\alpha \equiv N_{l} / N_{s}$ and $\beta \equiv\left(1-\bar{\phi}_{s}\right) / \bar{\phi}_{s}$. From this, one can show that the integrated excess of short polymers is

$$
\frac{\theta_{s}}{a \rho_{0}} \approx \frac{2 A(\alpha-1) \bar{\phi}_{s}\left(1-\bar{\phi}_{s}\right)}{\alpha-(\alpha-1) \bar{\phi}_{s}}
$$

which coincides with eq 1 for the special case of a bidisperse melt.

\section{Results}

We begin by considering monodisperse melts. Figure 1a plots the average concentration profile, $\phi(z)$, from simulations for $N=80$ with and without the external field, $w(z)$, which is shown in the inset. Figure $1 \mathrm{~b}$ goes on to compare the end-monomer concentration, $\phi_{e}(z)$, from the simulation (solid curve) to that of mean-field theory (dashed curve). The agreement is remarkably good. In both cases, there is a substantial excess of end monomers next to the wall (i.e. $z=0$ ), followed by a depletion that extends far into the melt. The amplitude of the

depletion must balance the excess at the surface due to the conservation of end monomers, 
and consequently the amplitude depends on the model details. On the other hand, the depletion is predicted to exhibit the universal shape in eq 17, with a range that scales as $a \sqrt{N}$
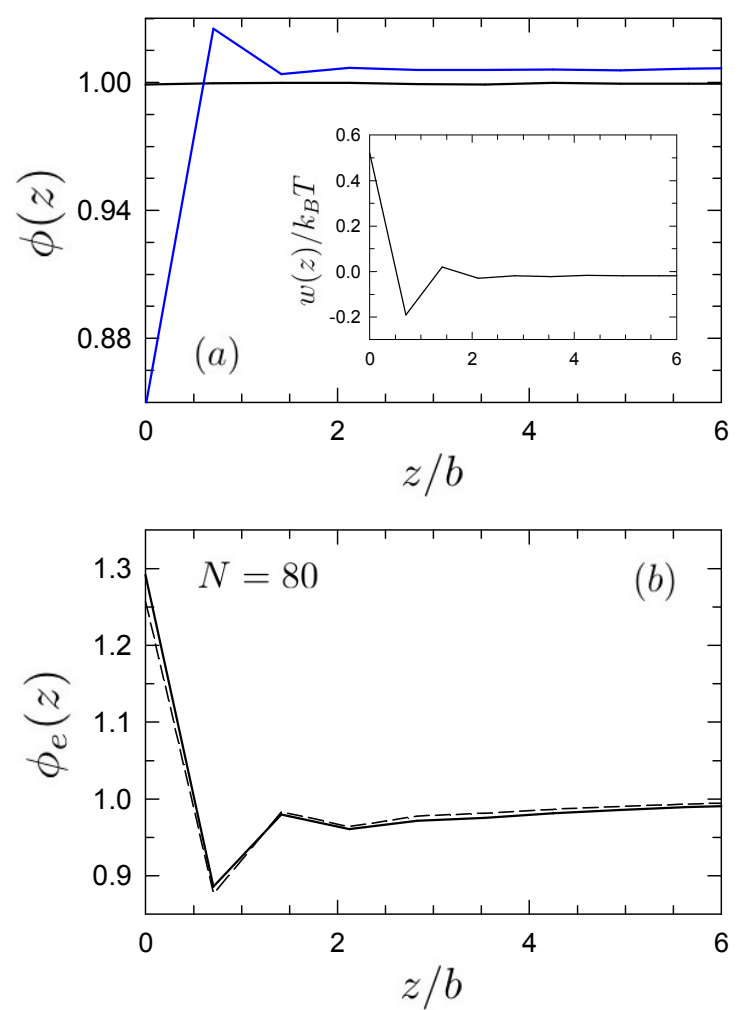

Figure 1: (a) total concentration, $\phi(z)$, of monodisperse $N=80$ polymers from simulations without (blue curve) and with (black curve) an external field, $w(z)$. The field producing the step profile is shown in the inset. (b) distribution of end monomers, $\phi_{e}(z)$, from the simulation for the step profile (solid curve) compared to mean-field theory (dashed curve).

Figure 2a zooms in on the end-monomer depletion from the simulations (solid curves) and mean-field theory (dashed curves) for three different polymerizations, $N$. As expected, the range of the depletion increases for larger polymers. Although the simulations agree reasonably well with mean-field theory, the range of the depletion is noticeably larger in the simulations. However, recall that the segment length in the simulations is $a=1.233 b$, whereas in mean-field theory it is simply $a=b$. The increased segment length is largely due to the fact that chain back-folding is prohibited in the simulations. As we can see from Figure $2 \mathrm{~b}$, when the scaled excess, $\delta \phi_{e}(z) \sqrt{N}$, is plotted in terms of $z / a \sqrt{N}$, both 
the simulation and mean-field results roughly collapse onto the universal prediction, eq 17, from linear-response theory (red curve). The small deviations from a perfect collapse can be largely attributed to the finite chain lengths, $N$.
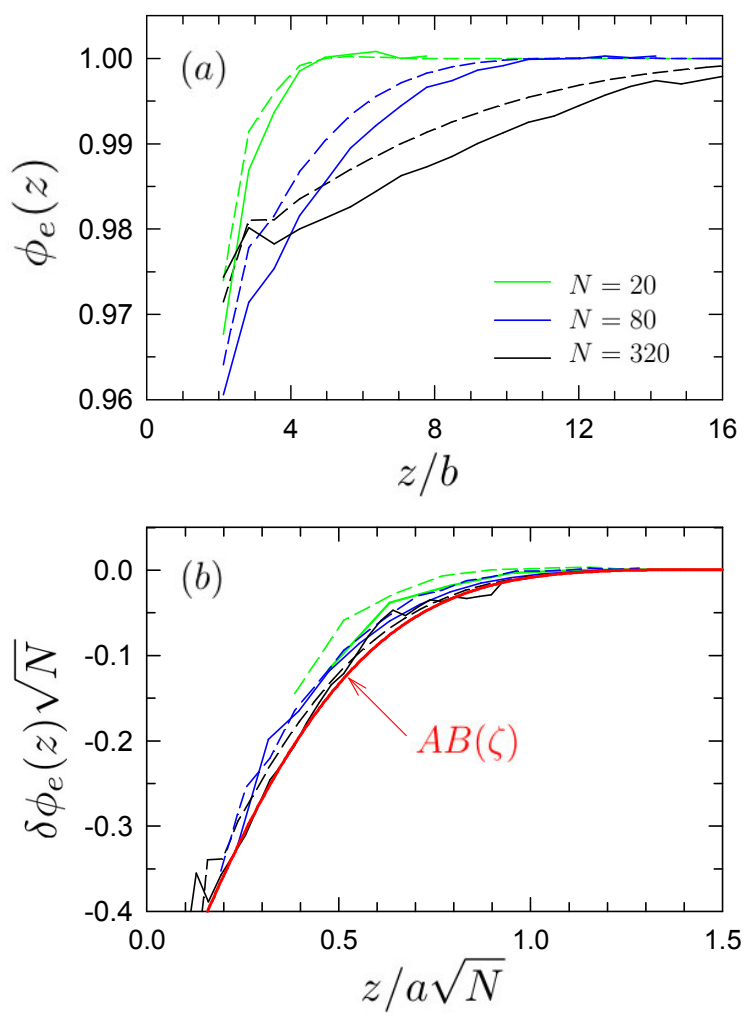

Figure 2: (a) depletion of end monomers in monodisperse melts of various polymerizations, $N$, obtained from simulation (solid curves) and mean-field theory (dashed curves). (b) scaling plot comparing the results to eq 17 from linear-response theory (red curve).

We now move on to the bidisperse melts. Figure 3a compares the concentration of short polymers, $\phi_{s}(z)$, from simulation (solid curves) to that of mean-field theory (dashed curves) for different degrees of polymerization. As the polymerization increases, the amplitude of the segregation decreases while the range increases. In all cases, the simulation and meanfield results are reasonably similar, but the amplitude and the range are somewhat larger in the simulations. Figure $3 \mathrm{~b}$ compares the scaled results to the prediction, eq 20, from linear-response theory (red curve). Again, the difference in segment lengths accounts for the difference in the range of the segregation, but the difference in amplitude still remains. Figure 3c compares the integrated excesses, $\theta_{s}$, to the prediction, eq 24 , from linear-response theory 

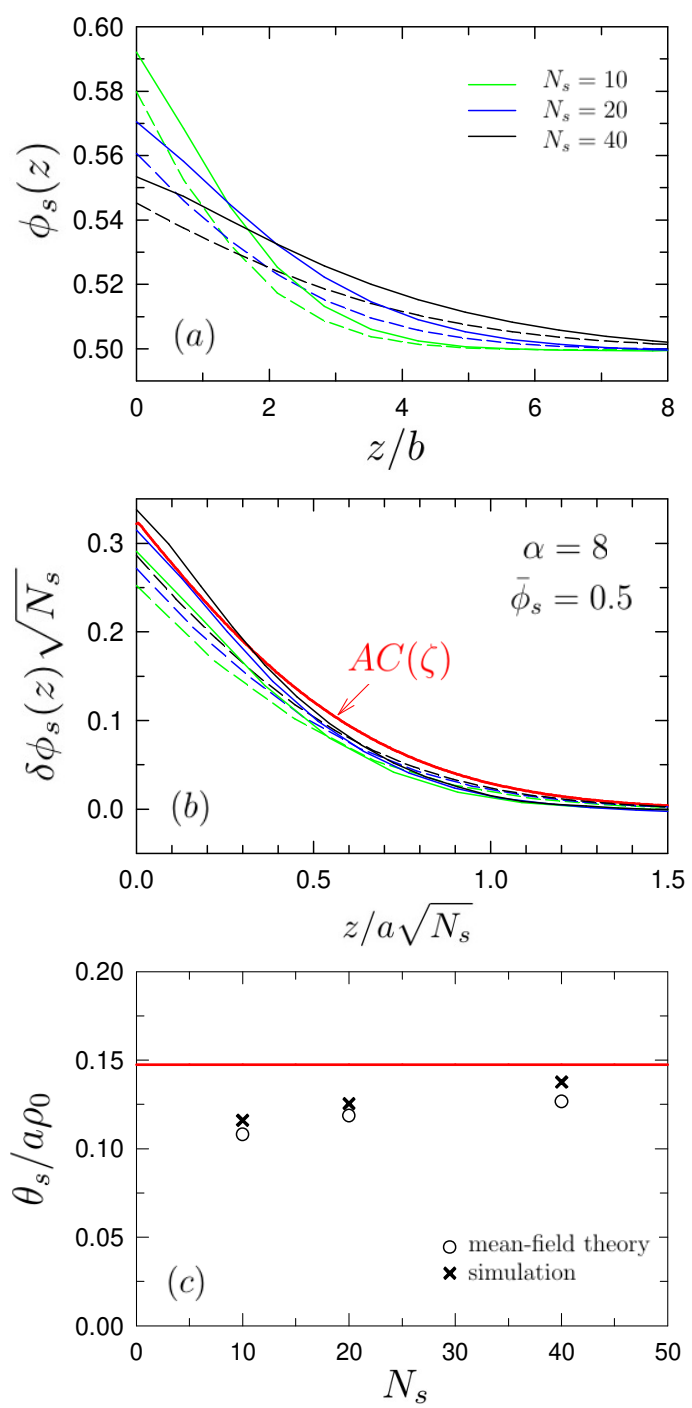

Figure 3: (a) concentration of short polymers, $\phi_{s}(z)$, for bidisperse melts of different polymerizations with fixed $\alpha \equiv N_{l} / N_{s}=8$ and $\bar{\phi}_{s}=0.5$, obtained from simulation (solid curves) and mean-field theory (dashed curves). (b) scaling plot comparing the results to eq 20 from linear-response theory (red curve). (c) integrated excess of short polymers, $\theta_{s}$, from simulations (crosses) and mean-field theory (circles) compared to eq 24 from linear-response theory (red line). 
(red line). As observed previously, ${ }^{18}$ the mean-field results approach the linear-response prediction from below as $N_{s}$ approaches infinity. The simulation results are slightly above the mean-field predictions, reflecting the elevated amplitudes observed in Figure $3 \mathrm{~b}$.
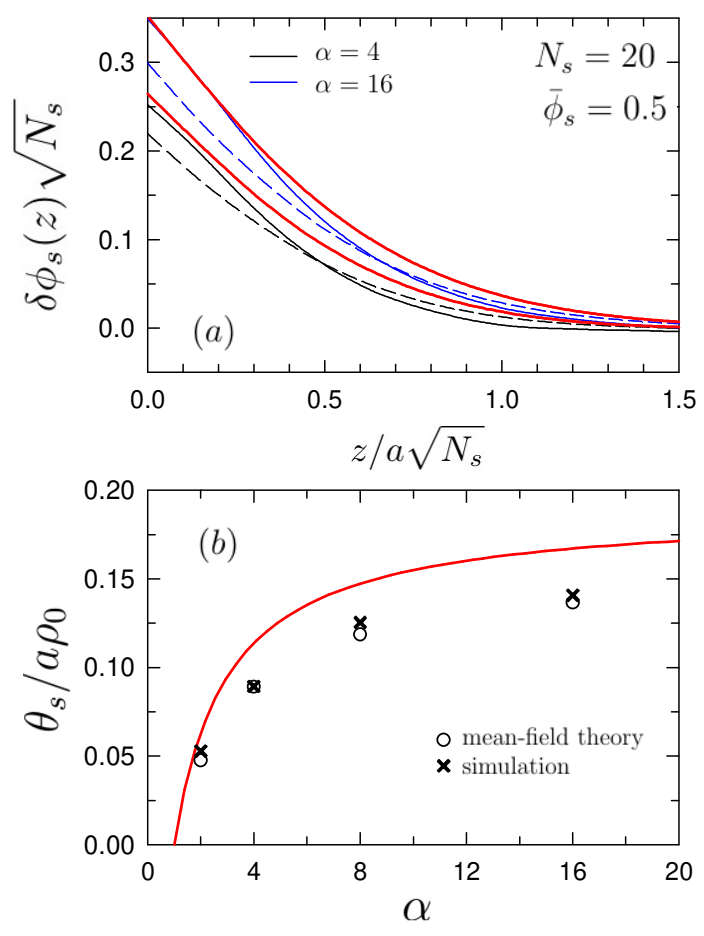

Figure 4: (a) scaling plot for the excess concentration of short polymers in a bidisperse melt for $N_{l}=80$ and 320 with fixed $N_{s}=20$ and $\bar{\phi}_{s}=0.5$, obtained from simulation (solid curves) and mean-field theory (dashed curves). (b) integrated excess from simulation (crosses) and mean-field theory (circles). Red curves denote predictions from linear-response theory.

Next, we examine the accuracy of the mean-field theory for different ratios of polymerization, $\alpha=N_{l} / N_{s}$. Figure 4a starts by comparing the scaled excess concentration of short polymers for $N_{l}=80$ and 320 , with fixed values of $N_{s}=20$ and $\bar{\phi}_{s}=0.5$. As before, the simulation (solid curves) and the mean-field (dashed curves) results are similar, but the amplitude of the segregation is somewhat greater in the simulation. This is also evident in Figure $4 \mathrm{~b}$, where the integrated excess, $\theta_{s}$, is plotted as a function of $\alpha=N_{l} / N_{s}$. As we can see, $\theta_{s}$ approaches zero as $\alpha$ approaches one, which must be the case since this limit corresponds to a monodisperse melt. Although the linear-response predictions (red curves) are in reasonable agreement with the simulation and mean-field results, they are not particularly 
accurate. This is undoubtedly because of the relatively low polymerization $N_{s}=20$.
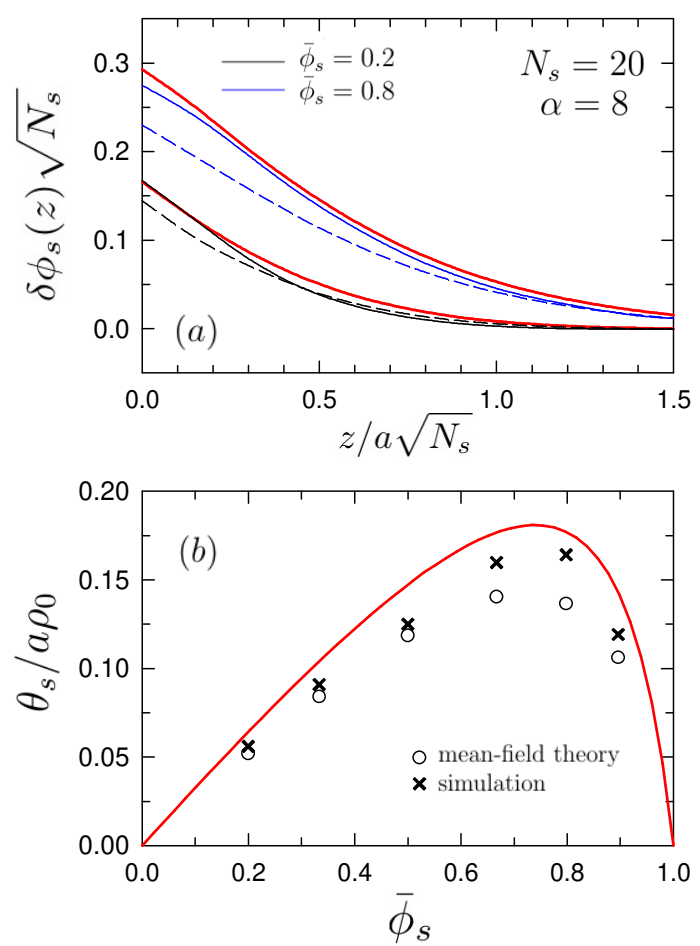

Figure 5: Analogous plots to those of Figure 4 showing the dependence on $\bar{\phi}_{s}$ for $N_{s}=20$ and $N_{l}=160$.

Lastly, we check the agreement between simulation and mean-field theory over a range of compositions. Figure 5a compares predictions of the excess concentration for $\bar{\phi}_{s}=0.2$ and 0.8 , with fixed polymerizations of $N_{s}=20$ and $N_{l}=160$. There is good agreement for the lower composition, but less so for the higher one. Figure 5b compares the integrated excess, $\theta_{s}$, from simulation and mean-field theory as a function of $\bar{\phi}_{s}$. Naturally, the segregation has to vanish in the limits $\bar{\phi}_{s}=0$ and 1 , since they correspond to monodisperse melts. The position of maximum segregation is consistent with the linear-response prediction, $\bar{\phi}_{s}^{\max }=$ $1 /\left(1+\alpha^{-1 / 2}\right)=0.739$. However, the integrated excess predicted by mean-field theory proves to be least accurate near the peak, underestimating the simulations by up to $20 \%$. The linearresponse predictions (red curves) agree better with the simulations than the full mean-field theory, but that is only because of a compensating inaccuracy due to the relatively low value of $N_{s}=20$. 


\section{Discussion}

The aim of this study was to assess the inaccuracy in the mean-field predictions for entropic surface segregation resulting from excluded-volume effects. In order to isolate the effects, it was crucial that the simulations and mean-field theory were performed using the exact same model. We also needed to obtain accurate statistics for relatively long chains (e.g., $N \approx 300$ ), and therefore we chose the simplest model conceivable. Using an external field, $w(z)$, to control the shape of the surface profile also helped isolate the effects and speed up the simulations. Although it would have been feasible to produce the surface using appropriate interactions rather than a field, that would have resulted in nontrivial concentration profiles that would have only complicated the comparison with mean-field theory. By taking our simpler approach, the simulations were exactly equivalent to the mean-field calculation, apart from the fact that the simulations rigorously respected the excluded-volume interaction by preventing more than one monomer per lattice site.

There was good reason to expect the simulations to agree with the universal behaviors, $\delta \phi_{e}(z) \propto B(z / a \sqrt{N})$ and $\delta \phi_{s}(z) \propto C\left(z / a \sqrt{N_{s}}\right)$, predicted by linear-response theory, despite the fact that it relies on the mean-field approximation. This is because these linear-response predictions are independent of the short-range details of the system. In the bulk, we know that the excluded-volume interactions are screened on the molecular length scale, ${ }^{1}$ which is why polymers obey random-walk statistics and thus why mean-field theory is accurate for polymer melts. The agreement we observed simply implies that this screening of the excluded-volume interactions continues to hold in the surface region of the melt.

Although we could have anticipated the agreement with $B(\zeta)$ and $C(\zeta)$, there was no way to know that the amplitude of the segregation would agree so well with the prediction, $A$, in eq 16 . The amplitude is influenced by the short-range details of the model, and thus excluded-volume interactions could still affect $A$ despite the screening. Indeed, we have previously pointed out that the excluded-volume interactions cause violations to the Silberberg argument, and therefore could contribute significantly to the entropic segregation. ${ }^{14}$ How- 
ever, as it turns out, the present study finds that the effect is relatively minor, at least, for our simple lattice model. It would be useful to repeat the comparison between simulation and mean-field theory for other, perhaps more realistic, models in order to test the generality of this finding.

In simulations, the interactions between all the monomers are usually identical, which ensures that the surface segregation is entirely due to entropic effects. However, in real systems, the interactions of end monomers will always be slightly different from those of middle monomers, which will produce an effective enthalpic interaction between chain ends and the surface. ${ }^{23}$ This enthalpic effect may very well dominate the entropic one. Nevertheless, the linear-response calculation remains the same when these enthalpic interactions are included, apart from an additional contribution to the amplitude, $A \cdot{ }^{17}$ Thus, the universal functions, $B(\zeta)$ and $C(\zeta)$, should still apply to actual experiments.

\section{Summary}

We have tested mean-field predictions for the entropic enrichment of chain ends to the surface of a monodisperse melt and of short polymers to the surface of a bidisperse melt. This was done by performing simulations and mean-field calculations on the exact same lattice model. The excluded-volume interactions, which are treated properly by the simulations but not the mean-field theory, cause a modest increase in the statistical segment length, $a$. The shape of the long-range depletion of chain ends that balances the enrichment agrees with the universal function, $B(z / a \sqrt{N})$, predicted by mean-field theory once the change in segment length is accounted for. Likewise, the enrichment of short polymers in the bidisperse melt matched the universal shape, $C\left(z / a \sqrt{N_{s}}\right)$. These findings are consistent with the notion that excluded-volume interactions are screened on molecular length scales. Although there is no $a$ priori reason to expect mean-field theory to accurately predict the non-universal (i.e., modeldependent) amplitude, $A$, of the entropic segregation, it only underestimated the effect by a 
small amount. Thus, mean-field theory appears to provide reliable predictions for polymer configurations at surfaces.

\section{ORCID}

Pendar Mahmoudi: 0000-0001-7280-5594

Thomas M. Beardsley: 0000-0002-6551-7866

Mark W. Matsen: 0000-0003-2099-0892

\section{Acknowledgments}

This work was supported by the Natural Science and Engineering Research Council (NSERC) of Canada.

\section{References}

(1) Wittmer, J.P.; Cavallo, A.; Xu, H.; Zabel, J.E.; Poliüska, P.; Schulmann, N.; Meyer, H.; Fatago, J.: Johner, A.; Obukhov, S.P.; Baschnagel, J. Scale-Free Static and Dynamical Correlations in Melts of Monodisperse and Flory Distributed Homopolymers: A Review of Recent Bond-Fluctuation Model Studies J. Stat. Phys. 2011, 145, 1017-1126.

(2) Silberberg, A. Distribution of Conformations and Chain Ends near the Surface of a Melt of Linear Flexible Macromolecules. J. Colloid Interface Sci. 1982, 90, 86-91.

(3) Skortsov, A.M.; Leermakers, F.A.M.; Fleer, G.J. Equivalence of Chain Conformations in the Surface Region of a Polymer Melt and a Single Gaussian Chain Under Critical Conditions. J. Chem. Phys. 2013, 139, 054907.

(4) Sarabadani, J.; Milchev, A.; Vilgis, T.A. Structure and Dynamics of Polymer Melt 
Confined Between Two Solid Surfaces: A Molecular Dynamics Study. J. Chem. Phys. 2014, 141, 044907.

(5) Kumar, S.K.; Vacatello, M.; Yoon, D.Y. Off-Lattice Monte Carlo Simulations of Polymer Melts Confined between Two Plates. J. Chem. Phys. 1988, 89, 5206-5215.

(6) Bitsanis, I.; Hadziioannou, G. Molecular Dynamics Simulations of the Structure and Dynamics of Confined Polymer Melts. J. Chem. Phys. 1990, 92, 3827-3847.

(7) Kumar, S.K.; Vacatello, M.; Yoon, D.Y. Off-Lattice Monte Carlo Simulations of Polymer Melts Confined between Two Plates. 2. Effects of Chain Length and Plate Separation. Macromolecules 1990, 23, 2189-2197.

(8) Yethiraj, A.; Hall, C.K. Monte-Carlo Simulation of Polymers Confined Between Flat Plates. Macromolecules 1990, 23, 1865-1872.

(9) Pai-Panandike, R.S.; Dorgan, J.R.; Pakula, T. Static Properties of Homopolymer Melts in Confined Geometries Determined by Monte Carlo Simulation. Macromolecules 1997, 30, 6348-6352.

(10) Daoulas, K.C.; Harmandaris, V.A.; Mavrantzas, V.G. Detailed Atomistic Simulation of a Polymer Melt/Solid Interface: Structure, Density, and Conformation of a Thin Film of Polyethylene Melt Adsorbed on Graphite. Macromolecules 2005, 38, 5780-5795.

(11) Müller, M.; Steinmüller, B.; Daoulas, K.C.; Ramírez-Hernández, A.; de Pablo, J.J. Polymer-Solid Contacts Described by Soft, Coarse-Grained Models. Phys. Chem. Chem. Phys. 2011, 13, 10491-10502.

(12) Wu, D.T.; Fredrickson, G.H.; Carton, J.-P.; Ajdari, A.; Leiber, L. Distribution of Chain Ends at the Surface of a Polymer Melt: Compensation Effects and Surface Tension. J. Polym. Sci., Part B 1995, 33, 2373-2389. 
(13) Matsen, M.W.; Mahmoudi, P. Segregation of Chain Ends to the Surface of a Polymer Melt. Eur. Phys. J. E 2014, 37, 78.

(14) Mahmoudi, P.; Matsen, M.W. Segregation of Chain Ends to the Surface of a Polymer Melt: Effect of Surface Profile versus Chain Discreteness. Eur. Phys. J. E 2016, 39, 78.

(15) Hariharan, A.; Kumar, S.K.; Russell, T.P. A Lattice Model for the Surface Segregation of Polymer Chains Due to Molecular Weight Effects. Macromolecules 1990, 23, 35843592.

(16) Van der Gucht, J.; Besseling, N.A.M.; Fleer, G.J, Surface Segregation in Polydisperse Polymer Melts. Macromolecules 2002, 35, 6732-6738.

(17) Minnikanti, V.S.; Qian, Z.; Archer, L.A. Surface Segregation and Surface Tension of Polydisperse Polymer Melts. J. Chem. Phys. 2007, 126, 144905.

(18) Mahmoudi, P.; Matsen, M.W. Entropic Segregation of Short Polymers to the Surface of a Polydisperse Melt. Eur. Phys. J. E 2017, 40, 85.

(19) Vassiliev, O.N.; Matsen, M.W. Fluctuation Effects in Block Copolymer Melts. J. Chem. Phys. 2003, 118, 7700-7713.

(20) Glaser, J.; Medapuram, P.; Beardsley, T.M.; Matsen, M.W.; Morse, D.C. Universality of Block Copolymer Melts. Phys. Rev. Lett. 2014, 113, 068302.

(21) Scheutjens, J . M. H. M.; Fleer, G. J. J. Statistical Theory of the Adsorption of Interacting Chain Molecules. 1. Partition Function, Segment Density Distribution, and Adsorption Isotherms. Phys. Chem. 1979, 83, 1619-1635.

(22) Scheutjens, J . M. H. M.; Fleer, G. J. J. Statistical Theory of the Adsorption of Interacting Chain Molecules. 2. Train, Loop, and Tail Size Distribution. Phys. Chem. 1980, $84,178-190$. 
(23) de Gennes, P.G. Surface Tension of Molten Polymers. C.R. Acad. Sci. Paris 1988, 30\%, $1841-1844$. 


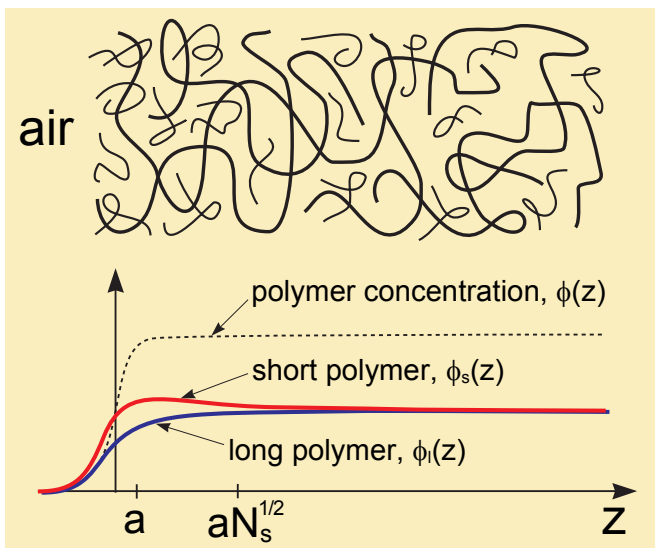

for Table of Contents use only

Title: Testing the Universality of Entropic Segregation at Polymer Surfaces

Authors: P. Mahmoudi, W.S.R. Forrest, T.M. Beardsley, and M.W. Matsen 